\title{
The extraretinal signal from the pursuit-eye-movement system: Its role in the perceptual and the egocentric localization systems
}

\author{
HITOSHI HONDA \\ Niigata University, Niigata, Japan
}

\begin{abstract}
The accuracy of perceptual judgment of the distance of a moving target tracked at various velocities by pursuit eye movements was examined in relation to the amount of two types of eye movement (smooth pursuit eye movement and compensatory saccade) involved in eye tracking. The perceptually judged distance became shorter as the amount of pursuit-eye-movement component in eye tracking increased. A detailed analysis of the eye-movement data and the size of perceptual underestimation indicated that the underestimation was mainly caused by inaccurate extraretinal information derived from the pursuit-eye-movement system, which underestimated the distance at a constant ratio, irrespective of the velocity of tracking. Egocentric localization was not affected by the mode of eye movements, indicating that the egocentric localization system functions without interference from the inaccurate information from the pursuit-eye-movement system.
\end{abstract}

It is well known that when a moving visual target is tracked in the dark by a pursuit eye movement (PEM), the observer's perception of the target's movement differs from its actual movement. For example, an object moving at a certain velocity appears to be moving faster when it is observed with the eyes stationary than when it is visually pursued (Aubert-Fleischel phenomenon). Festinger and Easton (1974) found that the perceived path of a moving light spot tracked by a PEM was not its actual path. Similarly, Mack and Herman (1972) and Honda (1985) reported that when a moving target was tracked by a PEM, the target's distance was judged to be shorter than it was when scanned by a saccade. According to the cancellation theory of visual stability, perception at the time of eye movements in the dark is determined on the basis of (1) information about the position of visual stimuli on the retina-that is, retinal signal (RS) - and (2) information about the eye position in the orbit derived from the oculomotor systemthat is, extraretinal signal (ERS). Therefore, the findings described above have been interpreted as showing that the oculomotor system for PEMs does not provide an accurate ERS about eye position to the perceptual system. Stoper (1973) drew a similar conclusion in his study on apparent movement of stimuli successively presented during PEMs.

However, it is also well known that in eye tracking, especially when the velocity of the target is high, PEMs

This research was supported by a 1985 Grant-in-Aid for Scientific Research (59510052) from the Japanese Ministry of Education, Science, and Culture to the author. I am grateful to Arien Mack, Myron L. Braunstein, and two anonymous reviewers for their useful comments on an earlier draft of the manuscript. The author's mailing address is: Department of Psychology, Faculty of Humanities, Niigata University, Ikarashi, Niigata 950-21, Japan. are frequently interrupted by compensatory small saccades (Collewijn \& Tamminga, 1984). Compensatory saccades during PEMs occur when the eye lags behind the target and when, immediately before the compensatory saccades, the image of the target is not on the fovea. Therefore, compensatory saccades provide RSs concerning the discrepancy between the eye and the target. This means that as the saccade component in eye tracking increases, the subjects are given fewer ERSs from the PEM system (pursuit ERS), but given more RSs and ERSs from the saccade system.

In Mack and Herman's (1972) and Honda's (1985) studies, however, no analysis was made of the amount of compensatory saccades involved in PEM tracking. Therefore, these studies do not seem to present a conclusive result regarding the size of perceptual underestimation in judging the distance of a target's movement tracked by a PEM. The present study was designed to solve this problem. To this purpose, I employed three kinds of target velocity, expecting that compensatory saccades increase with the target's velocity, and asked subjects to reproduce the perceptually judged distance of the target's movement that they tracked. It was expected that subjects underestimate the distance less as compensatory saccades increase, because these saccades provide the perceptual system with both the RS and the ERS from the saccade system. To confirm this prediction, a detailed analysis was made of the relation between the size of perceptual underestimation and the types of eye movement (compensatory saccade and PEM) involved in visual tracking.

In Honda's (1985) study, in addition to perceptual judgment of the distances of targets' movement, the subjects were asked to locate egocentrically a target they tracked with a PEM, and the results showed that there is a substan- 
tial difference between these two types of visual processing. In that study, however, only two eye-movement conditions (saccade and PEM) were employed. I therefore replicated the experiment, using a target moving at various velocities, and attempted to confirm the results reported in Honda (1985).

\section{METHOD}

\section{Target Presentation}

The experiment was conducted in a dark room. Each subject was seated facing the apparatus for stimulus presentation and asked to hold the head firmly in the forehead-and-chin rest. A He-Ne laser beam (JHL-R20S, Nihon Lasers) was used as a visual target. The beam was projected on a black screen through a mirror mounted on a computer-driven galvanometer (PC-8000 system, NEC). A mirror was set before the subject's eyes, and the subject looked at the optical image of the laser spot $\left(0.2^{\circ}\right.$ in diameter, $\left.40 \mathrm{~cd} / \mathrm{m}^{2}\right)$ projected in front of the subject.

Visual targets were presented in five conditions (see Table 1). In Condition $S$ (step movement), an initial fixation point was presented for $2 \mathrm{sec}$ at the center of the visual field. At the offset of the fixation point, a visual target was presented for $400 \mathrm{msec}$ at a $13^{\circ}, 15.5^{\circ}$, or $18^{\circ}$ arc to the right of the fixation point. The subject moved the eyes by a saccade from the fixation point to the target position. It was expected that in Condition $S$, the subject would be given both the RS and the saccade ERS, because during the latency period (usually about $200 \mathrm{msec}$ ) of the saccade, the target image was projected on an eccentric position on the retina. In Condition FM (fast movement), $2 \mathrm{sec}$ after the onset of the fixation point, the target moved toward the right at a velocity of $20 \% \mathrm{sec}$ and stopped at one of the above-mentioned three target positions. The target was presented for $\mathbf{4 0 0} \mathrm{msec}$ at the final position. In Condition MM (moderate movement) and Condition SM (slow movement), a target was presented in the same way as in Condition FM, except that its velocity was $7 \% \mathrm{sec}$ (Condition MM) or $3 \% \mathrm{sec}$ (Condition SM). These three conditions (FM, MM, and SM) were employed to manipulate the amount of PEM component during eye tracking. In Conditions FM, MM, and SM, the subject was asked to track the moving target with a PEM as accurately as possible. In Condition $C$ (control), the procedure was the same as that for Condition S, except for the following modification: A scale was mounted horizontally across the black screen and illuminated by a small lamp. A fixation point and a visual target were presented on the scale. The target was presented for $2 \mathrm{sec}$, and the scale disappeared at the time of target offset. In Condition $\mathrm{C}$, therefore, the subject was given full knowledge of the actual position of the target. Condition $\mathrm{C}$ was employed separately for each subject, to discover constant directional bias in perceptual and motor responses.

\section{Perceptual Judgment and Egocentric Localization}

The subject's task was either to reproduce the apparent distance of the target displacement (perceptual judgment) or to point to the final target position with the unobservable right hand (egocentric localization).

In the perceptual judgment condition, after the buzzer warning, the target was presented in one of the five target-presentation conditions, and the subject made a saccade or tracked it with PEMs. One second after the offset of the target, two red LEDs $\left(0.2^{\circ}\right.$ in diameter, $25 \mathrm{~cd} / \mathrm{m}^{2}$ ) were presented for $6 \mathrm{sec}$. One LED, located $7^{\circ}$ to the left of the center of the visual field, was stationary, whereas the horizontal position of the other LED could be varied in $.5^{\circ}$ steps by rotating a knob. The subject's task was to reproduce the apparent distance of the target displacement by adjusting the distance between the two LEDs.

In egocentric localization, at the beginning of the trial, the subject placed the index finger of the unobservable right hand on a movable finger rest that was mounted on a rail placed along the path of the target displacement; it moved only in the horizontal direction. On each trial, a small yellow LED was turned on and off repetitively for $2 \mathrm{sec}$ to indicate the starting position of the pointing hand. The starting position was either near the initial fixation point $\left(3^{\circ}\right.$ to the right or $2^{\circ}$ to the left of the center of the visual field) or at the right side of the visual field $\left(21.5^{\circ}\right.$ or $26^{\circ}$ to the right of the center of the visual field). The subject moved the pointing hand to the indicated starting position. Two seconds after cessation of the yellow LED, the buzzer warning signal ( $2 \mathrm{sec}$ in duration) was given, and the target was then presented in one of the five targetpresentation conditions. The subject made a saccade to the target or tracked it with a PEM. After the offset of the target, the subject moved the pointing hand to the final target position. The subject was asked to keep watching the final target position while pointing. In Condition $\mathrm{C}$, in which a target was presented for $2 \mathrm{sec}$ at the final position on an illuminated scale, the subject pointed during its presentation. The position of the index finger of the pointing hand was monitored by a potentiometer connected to the finger rest.

Each subject was given eight sessions for each of the two response conditions. In each session, all combinations of three target positions and five target-presentation conditions-a total of 15 trialswere given in random sequence. The time interval between trials was about $7 \mathrm{sec}$. There was a rest period of about $5 \mathrm{~min}$ between sessions.

Before the experiment, each subject received a practice session of 15 trials for each response condition, without any feedback about performance.

\section{Eye Movement Recording}

The subject's horizontal eye movements were monitored by a photoelectric apparatus, which recorded the movement of the right eye with an accuracy to about $0.5^{\circ}$ for a $20^{\circ}$ rotation of the eye

Table 1

Comparison of Target-Presentation Conditions

\begin{tabular}{|c|c|c|c|c|}
\hline $\begin{array}{c}\text { Target-Presentation } \\
\text { Conditions } \\
\end{array}$ & $\begin{array}{c}\text { Movement } \\
\text { of Target }\end{array}$ & Background & $\begin{array}{l}\text { Duration at } \\
\text { Final Position } \\
\text { (in msec) }\end{array}$ & $\begin{array}{l}\text { Expected } \\
\text { Eye } \\
\text { Movements }\end{array}$ \\
\hline Condition S & steps & dark & 400 & saccade \\
\hline Condition FM & $\begin{array}{c}\text { fast movement } \\
\left(20^{\circ} / \mathrm{sec}\right)\end{array}$ & dark & 400 & pursuit \\
\hline Condition MM & $\begin{array}{l}\text { moderate movement } \\
\left(7^{\circ} / \mathrm{sec}\right)\end{array}$ & dark & 400 & pursuit \\
\hline Condition SM & $\begin{array}{c}\text { slow movement } \\
\left(3^{\circ} / \mathrm{sec}\right)\end{array}$ & dark & 400 & pursuit \\
\hline $\begin{array}{c}\text { Condition C } \\
\text { (control) }\end{array}$ & steps & $\begin{array}{l}\text { structured } \\
\text { field with } \\
\text { a scale }\end{array}$ & 2,000 & saccade \\
\hline
\end{tabular}


to the right of the central gaze. The overall system bandwidth was determined by the recticoder. The frequency characteristic curve of the recticoder was almost linear up to $100 \mathrm{~Hz}$. At the beginning and end of each session, a calibration trial was inserted, on which nine visual stimuli were sequentially presented from the center to the right of the visual field in $2.5^{\circ}$ steps, and the subject was asked to gaze at the stimuli as accurately as possible.

\section{Subjects}

Three subjects, the author (H.H.) and 2 male university students (H.M. and B.L.), participated in the experiment. The student subjects had never been in eye-movement experiments and had no knowledge about the aim of this experiment. The author and 1 student subject each had a slight myopia and wore spectacles during the experiment.

\section{RESULTS}

\section{Eye Movements}

The subjects' eye movements were recorded on a magnetic tape, using a data recorder (RMG-5304, Nihon Kohden), and later drawn on a recording chart by a largeamplitude recticoder (RJG-4024, Nihon Kohden) at a speed of $100 \mathrm{~mm} / \mathrm{sec}$. Analysis of eye movements was made through visual inspection of the chart and, if necessary, with a digital storage scope (DS-6121A, Iwatsu).

When the subjects were asked to make a saccade (Condition S), they failed in fixating the target with a saccade on 5 out of 144 trials (3.5\%) for the 3 subjects; that is, they moved their eyes before the target presentation. These trials were excluded from the data analysis of perceptual judgment and egocentric localization. The mean latencies of the saccades were $188 \mathrm{msec}(S D=17 \mathrm{msec})$, $197 \mathrm{msec}(S D=60 \mathrm{msec})$, and $186 \mathrm{msec}(S D=20 \mathrm{msec})$ for subjects H.H., H.M., and B.L., respectively. The duration of the saccades was approximately $30-50 \mathrm{msec}$. Thus, the subjects made a saccade during the target presentation $(400 \mathrm{msec})$.

In Conditions FM, MM, and SM, out of a total 432 trials for the 3 subjects, analysis of eye movements was impossible on 12 trials $(2.8 \%)$ because of recording failure. In the remaining 420 trials, the subjects' PEMs were frequently interrupted by saccades. On almost all trials of Condition FM, the subjects made two or three interrupting saccades of approximately $2^{\circ}-4^{\circ}$. Similarly, in Condition MM, their PEMs were interrupted by one, two, or three saccades of about $1^{\circ}-2^{\circ}$. In Condition SM, the size of the interrupting saccades was small (about $1^{\circ}$ ), and the frequency was different for each subject. Subjects H.M. and B.L. made one or two saccades per trial, whereas subject H.H.'s PEMs were rarely interrupted by a saccade. Calculation of the proportion of the target distance occupied by saccade components is exemplified in Figure 1. The proportions in each target-presentation condition are shown in Figure 2, separately for each subject. For all subjects, the percentage of the saccade component increased with increases in the target velocity.

\section{Perceptual Judgment}

Figure 3 shows the perceptually judged distance in five target-presentation conditions. The longitude in Figure 3

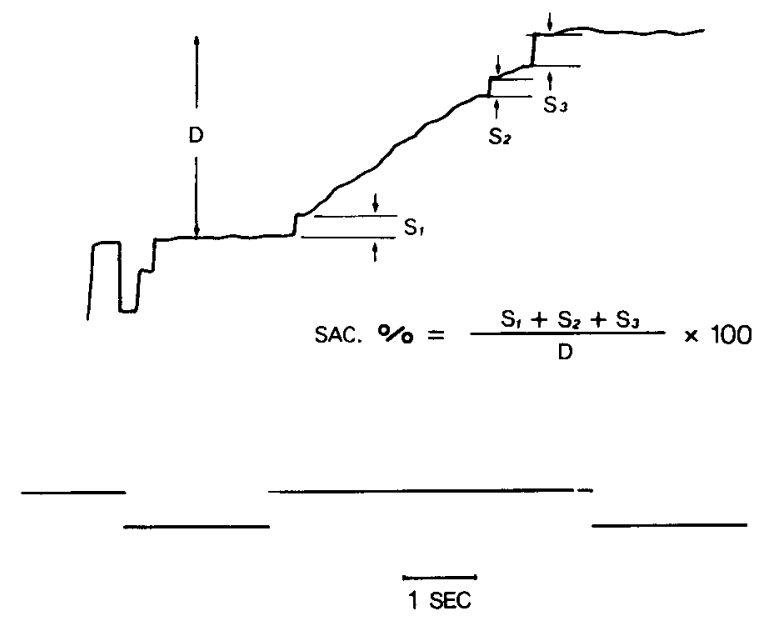

Figure 1. An example of calculation of the proportion of the target distance occupied by saccade components.

indicates the percentage of overestimation (plus) or underestimation (minus) of the distance. In Condition C, subjects showed overestimation from about 2\% (Subject H.M.) to $15 \%$ (Subject H.H.). Overestimation in Condition C can be interpreted as a constant directional bias, which was caused by the following factors in experimental method: the difference in light sources for the target (laser beam) and for perceptual judgment (LEDs), the time lag between the offset of the target and the onset of the LEDs, and the difference in spatial configuration between the path of the target displacement and the interval of the LEDs.

The perceptually judged distance gradually decreased as the velocity of the target decreased. According to statistical analysis, the difference in the perceived distance was significant among the five target-presentation conditions in all subjects $[F(4,115)=35.53, F(4,113)=17.32$, and $F(4,111)=14.88$, for Subjects H.H., H.M., and B.L., respectively, $p<.01$ in all cases]. A Tukey test showed no significant difference between Conditions $C$ and $S$ for two subjects, H.H. and N.M., but the difference was barely significant for Subject B.L. $(p=.05)$. For Subject H.H., the CE in Condition S was significantly different from the CEs in Conditions FM, MM, and SM. For Subjects H.M. and B.L., the CEs in Condition S were significantly different from those in Conditions $M M$ and SM. Significant differences were shown also between Conditions FM and SM (Subjects H.M. and B.L.) and between Conditions FM and MM (Subject H.M.).

In this experiment, as the target velocity increased, the proportions of subjects' tracking covered by saccades also increased and the durations of overall eye tracking decreased. Therefore, the results of the present study may be interpreted in terms of slower velocity or longer duration stimuli producing greater underestimation of moving distance.

To determine the primary source of underestimation, I analyzed the relation between the size of perceptual underestimation and the proportion of saccade component in- 

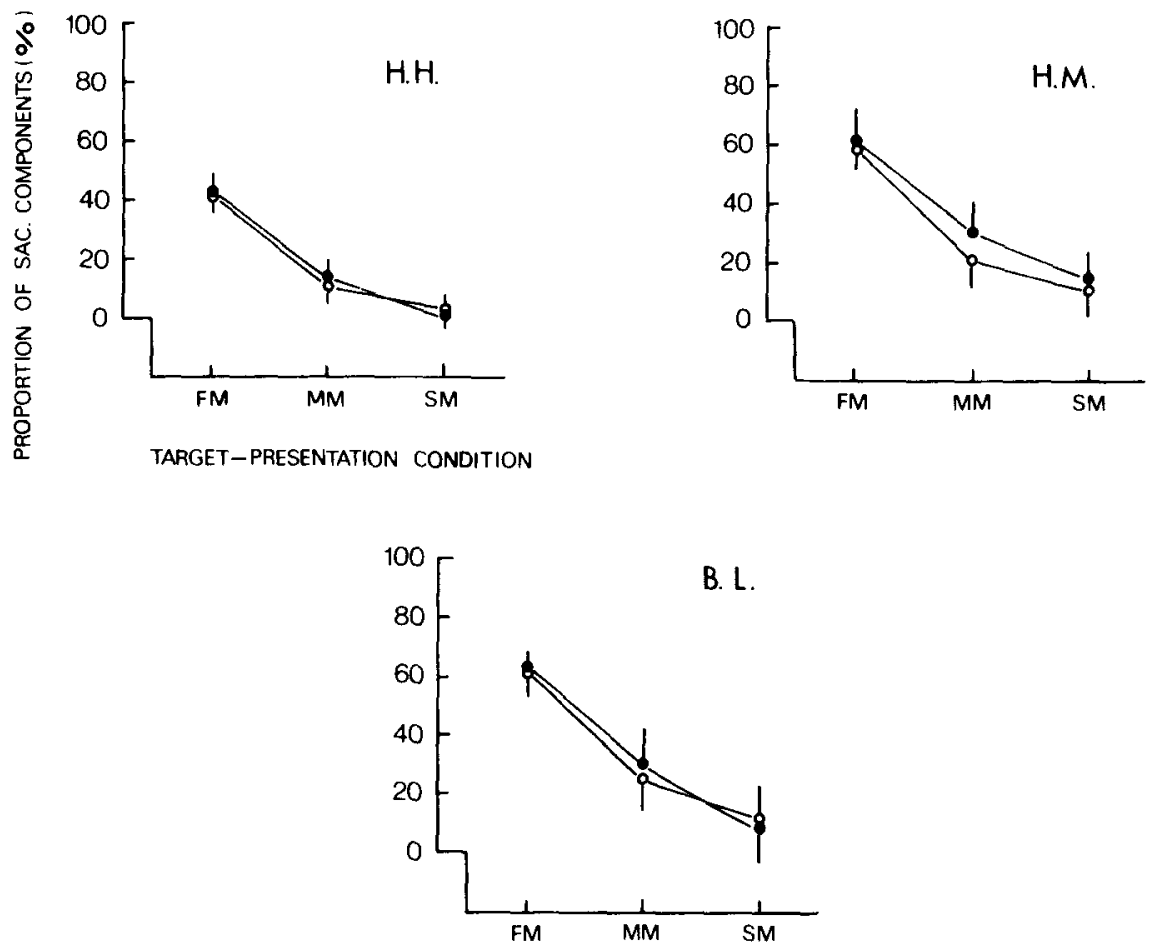

Figure 2. Proportion of saccade components for the three subjects. Open circles: results in egocentric localization. Filled circles: results in perceptual judgment.

volved in the subjects' tracking. As was described in the introduction, the saccades in eye tracking are thought to provide an RS about the discrepancy between the eye and the target. Therefore, we can assume that the distance of the target displacement scanned by saccades was correctly judged on the basis of the saccade RS, and that the underestimation of the distance was mainly caused by insufficient information from the pursuit ERS. In Table 2, the first $\left(D_{\text {sac }}\right)$ and second ( $\left.D_{\text {PEM }}\right)$ columns represent the proportions of target displacement covered by saccades and PEMs, respectively. The third column (PD) is the ratio of underestimation relative to estimation of distance based on saccadic observation (Condition S)-that is, the ratio obtained by dividing the perceived distance in each condition by that in Condition S. As already mentioned, the distance covered by saccades is assumed to be correctly estimated. Therefore, the forth column (PD $\left.-D_{\text {sac }}\right)$ represents the perceptual distance underestimated by the pursuit ERS. And the final column [(PD $\left.\left.-D_{\text {sac }}\right) / D_{\text {PEM }}\right]$ shows the efficiency (or gain) of the pursuit ERS in judging the distance of the target displacement. Note that for each subject, the efficiency of the pursuit ERS is almost the same across the three target-presentation conditions, indicating that the ratio of underestimation produced by the pursuit ERS is constant and independent of the velocity of eye tracking. Therefore, we can conclude that the size of underestimation increased with the proportion of PEM component involved in eye tracking, and that the velocity of eye tracking was not a critical factor.
Furthermore, there is no reason to believe that the results of the present study were caused by differences in the duration of eye tracking. If the duration of eye tracking had been involved in producing different perceptual distances in the present study, it would have been natural

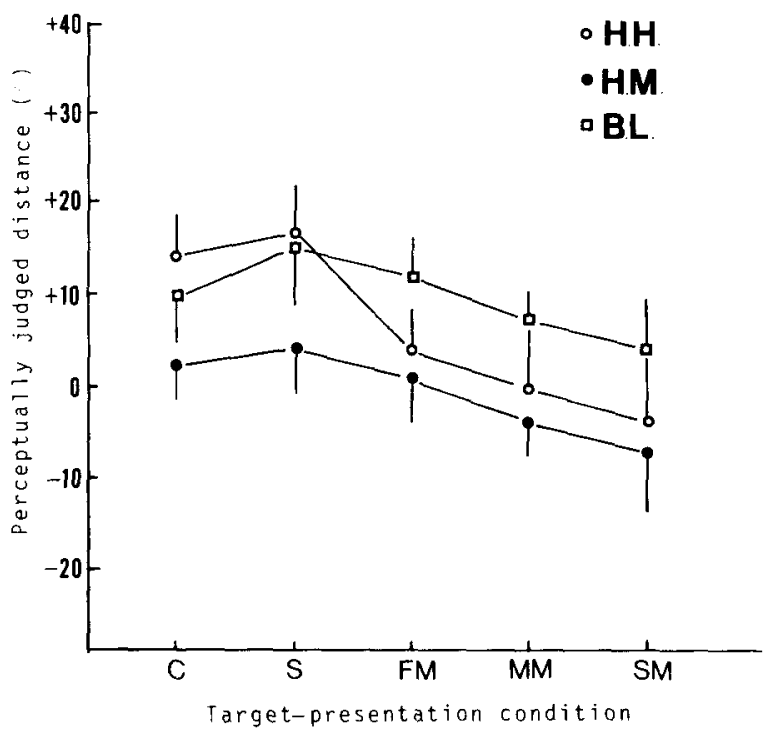

Figure 3. Perceptually judged distance of the target displacement for each subject. Plus and minus signs in the longitude indicate overestimation and underestimation, respectively. 
Table 2

Relation between Size of Perceptual Underestimation and Proportion of Saccade and PEM Components Involved in Eye Tracking

\begin{tabular}{ccccccc}
\hline & & & & & & $\left(\right.$ PD $\left.-D_{\text {sac }}\right) /$ \\
Subject & Condition & $D_{\text {sac }}$ & $D_{\text {PEM }}$ & PD & PD $-D_{\text {sac }}$ & $D_{\text {PEM }}$ \\
\hline H.H. & FM & 0.43 & 0.57 & 0.89 & 0.46 & 0.81 \\
& MM & 0.15 & 0.85 & 0.85 & 0.70 & 0.82 \\
& SM & 0.02 & 0.98 & 0.83 & 0.81 & 0.82 \\
H.M. & FM & 0.62 & 0.38 & 0.96 & 0.34 & 0.89 \\
& MM & 0.32 & 0.68 & 0.92 & 0.60 & 0.88 \\
& SM & 0.15 & 0.85 & 0.89 & 0.74 & 0.87 \\
B.L. & FM & 0.64 & 0.36 & 0.97 & 0.33 & 0.91 \\
& MM & 0.30 & 0.70 & 0.93 & 0.63 & 0.90 \\
& SM & 0.09 & 0.91 & 0.90 & 0.81 & 0.89 \\
\hline
\end{tabular}

Note $-D_{\text {sac }}=$ proportion of target displacement covered by saccades. $D_{\mathrm{PEM}}=$ proportion of target displacement covered by pursuit eye movements. $P D=$ ratio obtained by dividing the perceived distance in each condition by the distance based on saccadic observation (in Condition $S$ ). $\mathrm{PD}-D_{\mathbf{s a c}}=$ perceptual distance underestimated by the pursuit extraretinal signal. $\left(\mathrm{PD}-D_{\mathrm{sac}}\right) / D_{\mathrm{PEM}}=$ efficiency of the pursuit extraretinal signal in judging the distance of the target displacement.

to expect that longer duration of tracking would result in longer estimation of the tracked distance. However, this was not the case.

\section{Egocentric Localization}

The difference between the actual target position and the position pointed to by the subject was measured on each trial and used as a constant error (CE). Figure 4A shows the mean CEs in each target-presentation condition observed when the subject pointed from the right side. In Condition C, two subjects (H.H. and H.M.) localized the target at about the actual target position; that is, the CEs were about zero. On the other hand, Subject B.L. located the target at the right side of its actual position. The CEs in Condition $C$ were interpreted as a constant directional bias peculiar to each subject, caused mainly by a mismatch of information between the visual and the motor-output systems. A similar constant directional bias was observed when the subjects pointed from the center of the visual field (Figure 4B).

As is shown in Figure 4, when the subjects pointed from the center of the visual field, there was a substantial difference in CEs among the five target-presentation conditions; that is, as the velocity of the target movement became slower, the pointed positions shifted more toward the center of the visual field. On the other hand, the size of the difference in CEs was rather small in pointing from the right side. I statistically analyzed the results from each subject with a two-way ANOVA (5 target-presentation conditions $\times 2$ starting positions of the pointing hand). The main effect of starting position was significant for 2 subjects, reflecting the difference in constant directional
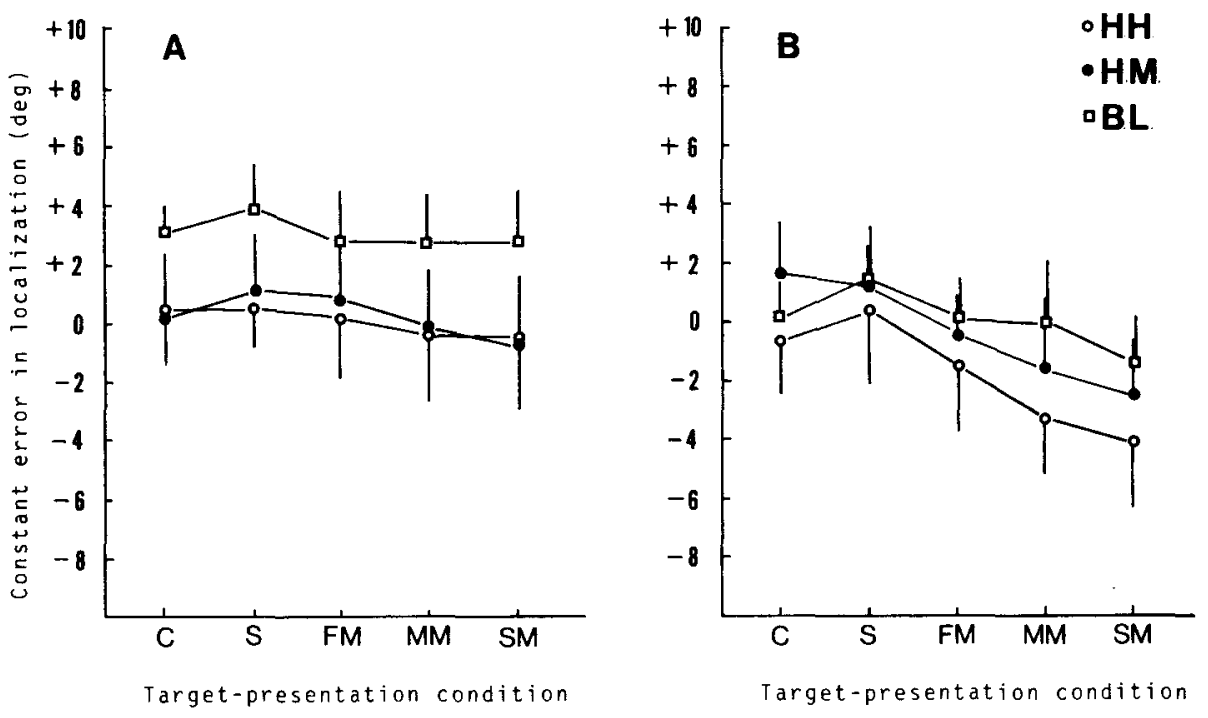

Figure 4. Mean constant errors (with standard deviations) in egocentric localization when subjects H.H., H.M., and B.L. pointed from the right side (A) and when they pointed from the center (B). Plus and minus signs in the longitude indicate the right and the left sides of the actual target position, respectively. 
bias between the two starting position conditions [Subject H.H., $F(1,110)=17.4$, Subject B.L., $F(1,110)=$ $132.2, p<.01$ in both cases]. As expected, the main effect of target-presentation conditions was significant in all subjects $[F(4,110)=5.17,7.16$, and 4.92 , for Subjects H.H., H.M., and B.L., respectively, $p<.01$ in all cases]. However, the interaction did not reach significance for any subject, indicating a possibility that in both starting position conditions, there was a tendency for the position localized by the hand to shift more toward the center of the visual field as the target velocity became slower. Next, I statistically examined the difference in the five target-presentation conditions for each starting position of the pointing hand. When the subjects pointed from the center, there was a significant difference in CEs among the five conditions $[F(4,110)=5.12,7.16$, and 5.45, for Subjects H.H., H.M., and B.L., respectively, $p<.01$ in all cases]. A subsequent Tukey test showed that the CEs in Condition $S$ were not different from those in Condition $\mathrm{C}$ for all subjects, but were significantly different from the CEs in Condition SM for all subjects and from those in Condition MF for Subjects H.H. and H.M. In contrast, when the subjects pointed from the right side, the difference in CEs among the five conditions was not significant for any subject.

\section{DISCUSSION}

\section{Perceptual Judgment and the Pursuit ERS}

The perceptually judged distance of the target displacement in Condition S was about the same as the perceptually judged distance in Condition $\mathrm{C}$, for all subjects. On the other hand, when the subjects tracked a moving target with PEMs, the distance perceived by the subjects became shorter in comparison to that in Conditions $S$ and $\mathrm{C}$ as the velocity of the target movement decreased. The result in perceptual judgment is, therefore, consistent with that reported by Mack and Herman (1972) and Honda (1985). However, an important finding was obtained by analyzing the relation between the size of perceptual underestimation and the amount of compensatory saccades involved in eye tracking.

As described in the introduction, the aim in this study was to explore the role of the RS and the saccade ERS in perceptual underestimation of the distance of a target's displacement tracked by PEMs. As a first step for this purpose, I examined the results obtained when the subjects made a saccade to the target, and found that there was almost no significant difference in perceived distance between Conditions $\mathbf{C}$ and $\mathrm{S}$. This indicates that, in judging the distance they scanned with saccades, the subjects could effectively use the RS and/or the saccade ERS.

On the other hand, when the subjects tracked the target movement with PEMs, they showed large underestimation in perceptual judgment, especially when the target moved slowly. Eye-movement data showed that the proportion of the PEM component decreased and that of the saccade component increased as the velocity of the target movement increased. Therefore, it seems that the pursuit ERS did not provide the subjects with useful information for judging the distance of the target displacement, and that in the three moving target conditions (FM, $M M$, and SM), the subjects judged the distance partly on the basis of the information from the interpolated compensatory saccades.

This speculation was supported by the analysis of the proportion of the saccade and the PEM components in eye tracking and their efficiency in judging the distance of the target displacement (Table 2). The results of the analysis gave strong support for the explanation that the perceptual underestimation was determined by the proportion of saccade and PEM components involved in eye tracking: The distance covered by saccades was correctly judged and the PEM system underestimated the distance it covered at a constant ratio independent of the tracking velocity, indicating that the PEM system does not provide the perceptual system with an accurate ERS about the distance the eye tracked.

As is shown in Table 2, however, the estimated size of underestimation of the PEM system differed between the 3 subjects. For Subjects H.M. and B.L., the size of underestimation was about $10 \%$, whereas the size was about $20 \%$ for Subject H.H. Although the reason for this between-subjects difference is not known at present, one possible reason might be that Subjects H.M. and B.L. had never been involved in eye-movement experiments before they participated in this study. It seems, therefore, that the 2 subjects failed in tracking the target smoothly with PEMs and showed many small compensatory saccades, which were overlooked in calculating the amount of saccade component involved in eye tracking. Therefore, if a precise analysis of compensatory saccades is possible, it may be shown that the size of underestimation of the PEM system is constant across subjects.

\section{Segregation of Perception and \\ Egocentric Localization}

The egocentric localization results confirmed the conclusion, reported by Honda (1985), that between the perceptual and the egocentric localization system there is a substantial difference in the role of pursuit ERS.

As is shown in Figure 4, when the subjects pointed to the target position from the center of the visual field, there was a large difference in the localized positions among the five target-presentation conditions. The localized position shifted more toward the left-that is, toward the position of the initial fixation point-as the velocity of the target movement decreased. The results of pointing from the center of the visual field (Figure 4B) were very similar to those of perceptual judgment (Figure 3). Honda (1985) explained this similarity by saying that pointing from the center of the visual field was affected by perceptual impression of the target movement, because the starting position and the direction of the target's movement were the same as those of the pointing hand: Instead of pointing at the egocentric position of the target, the 
subjects pointed at the target position by moving their hands through the perceived extent of the target displacement. Then, I performed the same analysis as is shown in Table 2 on the data from the localization task (pointing from the center). The estimated gains of pursuit ERS were, however, smaller than those obtained for the perceptual task, and not constant within each subject (Subject H.H., 0.5-0.75, Subject H.M., 0.73-0.78, Subject B.L., 0.81-0.89), indicating that the pointing from the center was not a simple copy of perceptual underestimation.

In contrast, when the subjects pointed from the right side, there was no difference in the located positions among the five target-presentation conditions. This indicates that the egocentric localization system functioned without interference from the inaccurate information about the prior PEM from the pursuit ERS, because even when a large part of their eye tracking was occupied by PEMs (Condition SM), the subjects located the target at about the same positions as located in Condition C.

One question here is how the egocentric localization system knew the exact target position in total darkness. There are two possible explanations. First, pursuit ERS has two kinds of information, information about the distance the eye tracked and information about the eye position; the egocentric localization system uses the latter and ignores the former. According to this explanation, the egocentric localization system knows the target position the eye tracked on the basis of eye position information derived from the pursuit ERS.

Second, it might be possible for subjects to point at the target position on the basis of the information from the current eye position, without needing to access any information from the PEM system. This explanation comes in part from the fact that in the present study the target remained visible for $400 \mathrm{msec}$ at the final position, and that during this period the subjects might get ready for orienting their responses to the absolute position of the target. This explanation seems very plausible. However, the information from the current eye position does not seem strong or sufficient enough to have lead the subjects to make an accurate localization, because the pointing from the center was evidently affected by a perceptual impression of the target movement even if the target remained visible for the same $400 \mathrm{msec}$ at the final position. Therefore, we cannot deny the possibility that the subjects pointed at the target position on the basis of the information about the eye position derived from the pursuit ERS. The existence of such eye position information was suggested by Hansen's (1979) study, in which the subjects accurately struck the position of a flashed target presented on a moving stimulus that they tracked with a PEM.

In any event, we can guess that the egocentric localization system functions without interference from the inaccurate information about the distance of the prior PEM. In other words, the system knows the direction of the eye, irrespective of the mode of the eye movement (i.e., saccade or PEM). The segregation of the egocentric localization system and the perceptual system was suggested by the idea of the "two visual systems" (Schneider, 1967; Treverthen, 1968) and has experimentally been demonstrated in many studies (Bridgeman, Kirch, \& Sperling, 1981; Bridgeman, Lewis, Heit, \& Nagle, 1979; Mack, Heuer, Fendrich, Villardi, \& Chambers, 1985; Mack, Heuer, Villardi, \& Chambers, 1985).

\section{REFERENCES}

Bridgeman, B., Kirch, M., \& Sperling, A. (1981). Segregation of cognitive and motor aspects of visual function using induced motion. Perception \& Psychophysics, 29, 336-342.

Bridgeman, B., Lewis, S., Heit, G., Nagle, M. (1979). Relation between cognitive and motor-oriented systems of visual position perception. Journal of Experimental Psychology: Human Perception \& Performance, 5, 692-700.

Collewiun, H. \& Tamminga, E. P. (1984). Human smooth pursuit and saccadic eye movements during voluntary pursuit of different target motions on different backgrounds. Journal of Physiology, 351, 217-250.

Festinger, L., \& EASTON, A. M. (1974). Inferences about the efferent system based on a perceptual illusion produced by eye movements. Psychological Review, 81, 44-58.

Hansen, R. M. (1979). Spatial localization during pursuit eye movements. Vision Research, 19, 1213-1221.

HondA, H. (1985). Spatial localization in saccade and pursuit-eyemovement conditions: A comparison of perceptual and motor measures. Perception \& Psychophysics, 38, 41-46.

Mack, A., \& Herman, E. (1972). A new illusion: The underestimation of distance during pursuit eye movements. Perception \& Psychophysics, 12, 471-473.

Mack, A., Heuer, F., Fendrich, R., Villardi, K., \& Chambers, D. (1985). Induced motion and oculomotor capture. Joumal of Experimental Psychology: Human Perception \& Performance, 11, 329-345.

Mack, A., Hever, F., Villard, K., \& Chambers, D. (1985). The dissociation of position and extent in Müller-Lyer figures. Perception \& Psychophysics, 37, 335-344.

SCHNEIDER, G. E. (1967). Contrasting visuomotor functions of tecrum and cortex in the golden hamster. Psychologische Forschung, 31, $52-62$.

STOPER, A. E. (1973). Apparent motion of stimuli presented stroboscopically during pursuit movement of the eye. Perception \& Psychophysics, 13, 201-211.

TreVerthen, C. B. (1968). Two mechanisms of vision in primates. Psychologische Forschung, 31, 299-337.

(Manuscript received November 20, 1989; revision accepted for publication June 18,1990 .) 